\title{
The Development of Direct Metallic Rapid Tooling System
}

\author{
Ren C. Luo, Fellow, IEEE, and Jyh Hwa Tzou
}

\begin{abstract}
In recent years, rapid tooling (RT) has become increasingly important because of the requirement of rapid manufacturing. RT can be used to produce temporary molds as well as permanent molds in mass production. RT provides more benefits from the design stage to mass production. The objective of this research is to develop the direct metallic RT system and identify process parameters of laser cladding. A $2.5-\mathrm{kW}$ Nd-AG laser system, a metallic powder feeder system, a lateral powder nozzle system, a PC-based motion controller, and a shielding gas-supply system are incorporated for the hardware architecture in the proposed RT system. The critical process parameters influencing the dimensional accuracy were analyzed with Taguchi's experimental design. From the analysis of variance computational results, the factors "Scanning speed" and "Tool path offset" are the influential factors affecting the layer thickness of RT parts. From the experimental results, the dimensional accuracy of the dual nozzle RT system is better than the single-nozzle RT system. Finally, the proposed RT system could fabricate the mold inserts. After finishing the mold inserts and assembling the plenary injection mold, the injection molding machine can generate acrylonitrile butadiene styrene plastic parts with excellent quality.
\end{abstract}

Note to Practitioners-Rapid prototyping (RP) technologies have provided a new way of making models or producing visualization prototype in a fast fashion. However, there is an urgent demand from the industry for much faster ways to make physical prototypes, with the desired material using the appropriate production method. RP technologies have introduced a new generation of RT processes. Producing tooling directly from computer-aided-design (CAD) models is regarded as an important process of reducing the cost and time to market for new products. In this paper, a direct metallic rapid tooling (RT) system is developed to fabricate the metallic parts and molds directly from 3-D CAD models. The experimental investigations are implemented to study the proposed RT system. Using Taguchi's method, the critical performance process parameters have been analyzed. The analysis of variance method is used to decide the important process parameters. The developed RT system can produce the mold inserts directly. After assembling the plenary injection mold, the Acrylonitrile butadiene styrene ABS plastic parts have been generated from this RT mold to demonstrate the success of the process. In the future, it is possible to develop a grading alloy RT system, which can control the ratio of the alloy grading layers. Because the grading alloy rapid mold can use varied metallic powders with varied ratio, the cooling time and the residual stress can be reduced. Furthermore, the RT system can also adopt the coaxial nozzle which can be integrated with the optical system. The advantage of the coaxial nozzle is the independence of the powder supply direction in which the workpiece moves.

Manuscript received April 13, 2005; revised August 23, 2005. This paper was recommended for publication by Associate Editor A. S. Kumar and Editor P. Ferreira upon evaluation of the reviewers' comments.

R. C. Luo is with the National Chung Cheng University, Chia-Yi 621, Taiwan, R.O.C. (e-mail: luo@ia.ee.ccu.edu.tw).

J. H. Tzou is with the Wu-Feng Institute of Technology, Chia-Yi 621, Taiwan, R.O.C. (e-mail: tzoujyh@mail.wfc.edu.tw).

Digital Object Identifier 10.1109/TASE.2006.872115
Index Terms-Injection mold, metallic, Nd-YAG laser, rapid tooling (RT), Taguchi's method.

\section{INTRODUCTION}

$\mathbf{R}$ APID PROTOTYPING (RP) is a modern technique that meets the current demand in the industry to reduce the design cycles, manufacturing time, and improve the design quality for new products. In recent years, rapid tooling (RT) has become increasingly important because of the high demand of rapid molds. RT can be defined as the use of RP as a tooling pattern for the purposes of molding production materials, or the direct production of a tool from the RP system. RT can be used to produce temporary molds as well as permanent molds in mass production. RT provides more benefits from the design stage to mass production [1].

Lasers have provided the industry with a new form of energy. The laser-based RT system is a significant advance in traditional RP techniques due to the direct fabrication of a near net shape component compared to the two-step process involving an intermediate step of mold preparation in conventional RP techniques. The two step process requires that 1) a pattern be made with RP techniques, from which 2) a mold is created. Laser-based RT systems have been developed to create functional, metallic prototypes with near net-shape geometries. The drawbacks for these systems are their low productivity and inability to consistently regulate component quality in terms of mechanical properties and geometry. To overcome these drawbacks, process control strategies have been utilized.

Some of the earlier attempts to build complex parts by layer addition were based on the laser cladding principle. Studies have been carried out to determine the effect of process parameters on the quality of the clad layer [2]. The quality of the clad layer is of importance as it forms the RT parts or molds. Another issue of concern is the fusion of layers to build the RT part. Kreutz et al. [3] has successfully developed some RT experiments based on these principles. Laser engineering net shaping (LENS) has demonstrated the feasibility to fabricate geometrically complex shapes with functional materials directly from a computer-aided design (CAD) model [4]. The integration of layered manufacturing and material removal processes is another developing alternative in rapid prototyping [5]. This enables the manufacture of RT parts with increased accuracy and surface finish.

Laser cladding is a material processing technique in which a laser is used as a heating source to melt the alloy powder to be cladded on to a substrate. With the laser cladding technique, an RT part can be fabricated by selective cladding layer by layer. The advantage of laser cladding is that through the 
melting of powder materials, it permits the generative manufacturing of a metallic part with a dense homogeneous structure. Direct light fabrication (DLF), which is being developed at Los Almos National Laboratory, has proven capable of producing metal parts with reasonably good accuracy and improved metallurgical properties [6]. Five axes of motion are used and the system can deliver up to four different powder compositions.

Laser direct cladding (LDC) and selective laser cladding (SLC) [7] are based on the same principle as laser cladding, where a gas jet containing fine metal powder is directed via a coaxial nozzle through the path of the laser beam, which is focused slightly above the workpiece. A small molten pool is formed on the surface of the workpiece where the molten particles land to form a layer upon cooling, about $0.5 \mathrm{~mm}$ thick, after the laser beam has moved on. LENS is the only current commercial direct metal fabrication system. The LENS system uses a focused Nd-YAG high-power laser to melt an area on a metal substrate while a nozzle simultaneously delivers metal powder to the molten pool. Direct metal deposition (DMD) is being used to fabricate molds and dies and for part repair. Mazumder et al. [8] demonstrated the application of laser-aided DMD to generate components with dimensional accuracy of 0.01 in with the required control of process parameters to obtain the desired microstructure.

Since the laser-based RT systems utilize the laser cladding principle to fabricate the RT part, the relevant parameters for the cladding process have to be studied carefully. These parameters play an important role in determining the clad profile, dilution of the cladding metal, fusion between layers, homogeneity of the layers, surface finish, defects such as porosity, cracking due to thermal stresses, plasma formation, etc. [9]. Dilution is an important factor for determining the thickness of the liquid layer on the substrate to the bonding of the current layer with the previous layer. In general, several parameters have to be varied simultaneously to obtain the desired characteristics of the deposited layer. Vetter et al. [10] performed experiments to determine the state of the powder when it arrives on the substrate surface. This forms an important limitation as the particles of the powder should be hot enough to adhere to the surface and form a layer, but not so hot that the particles vaporize and ionize to form plasma. The heat-affected zone (HAZ) induces surface distortion and residual stress and may be critical when producing small parts. In overlapping tracks, the process parameters can be varied in a defined range to obtain the required clad height and thickness which, in turn, are determined by bead geometry and an overlap factor. These parameters depend on the powder feed rate, power density, and computer numerical control (CNC) table feed rate. Care should be taken to avoid porosity, which occurs due to cavities between tracks that form from overlapped tracks or the evolution of entrapped gasses in the clad tracks. This can be avoided by a proper choice of overlap factor, which also determines the surface finish of the part. Laser based RT systems requires synchronization of three basic components of the laser deposition system, powder feeder system, energy delivery system, and CNC workstation. To improve the RT part quality, control of the variables of these systems is required.

The dimensional accuracy of the part depends on the uniformity and repeatability of the clad height and width being de-

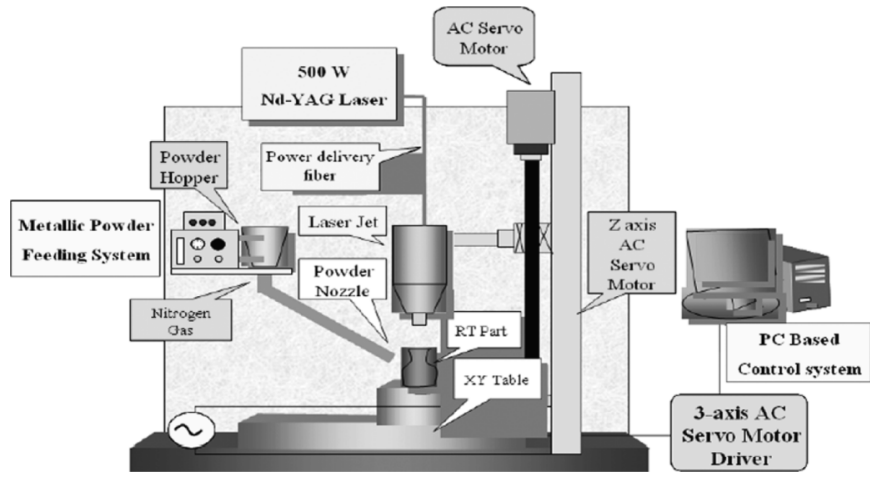

Fig. 1. Schematic architecture of the direct metallic RT system.

posited. Mazumder et al. [8] described the application of multiple sensors for closed-loop control of the bead height. The height controller shuts off the laser until it passes the excess buildup region. Another way to control the bead dimensions is by regulating the powder flow rate, provided the traverse speed of the CNC table is kept constant. Also, the carrier and shielding gas-flow rate can influence the amount of powder that gets deposited. By increasing the carrier and shielding gas-flow rate, the excess powder can be blown out of the way of the laser beam. A small deviation in mass flow rate results in large variations in the geometry and microstructure of the produced tracks.

\section{Hardware of the Direct Metallic RT System}

The hardware architecture of the direct metallic RT system is shown in Fig. 1. For the proposed RT process, the $2.5-\mathrm{kW}$ $\mathrm{Nd}-$ YAG laser beam is focused onto the substrate ( $\mathrm{S} 45 \mathrm{C}$ middle carbon steel) via a power delivery fiber, where it creates a molten pool while the lateral nozzle simultaneously delivers metallic powder to the substrate. The nickel-based alloy powder, which has better oxidation resistance, must be transported from the powder feeder to the molten pool. This can be done by the use of a carrier gas (argon, helium, and nitrogen) and by gravitation. Subsequently, the metallic powder is directed to the molten pool by one or two lateral powder nozzles. A four-axis digitial signal processor (DSP) motion controller is used to position the $\mathrm{X}-\mathrm{Y}$ table (workpiece and substrate) and the Z-axis (laser head). During the manufacturing process, the shielding gas (nitrogen) can protect the molten metal from oxygenation and protect the laser optics. The shielding gas can also restrain the metallic vapor and prevent ionization. Layer after layer, the metallic powder is added and fused to the previous layer until the RT part is completed [11], [12].

The direct metallic RT system can be described in terms of five subsystems: "Nd-YAG laser system," "Metallic powder feeder system," "3-axis working table," "Metallic deposition nozzle," and "Shielding gas supply system." These subsystems can be further introduced as follows:

\section{A. Nd-YAG Laser System}

There are two kinds of lasers to choose from continuous-wave (CW) laser and pulsed laser. We use the $\mathrm{CW}$ laser because it is stable, sustained, and high power. According to the difference in wavelength of the laser, at least two kinds of lasers- $-\mathrm{CO}_{2}$ lasers and Nd-YAG lasers-can be used in the laser cladding 


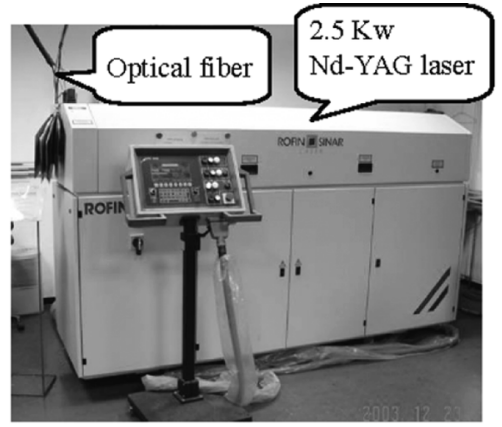

(a)

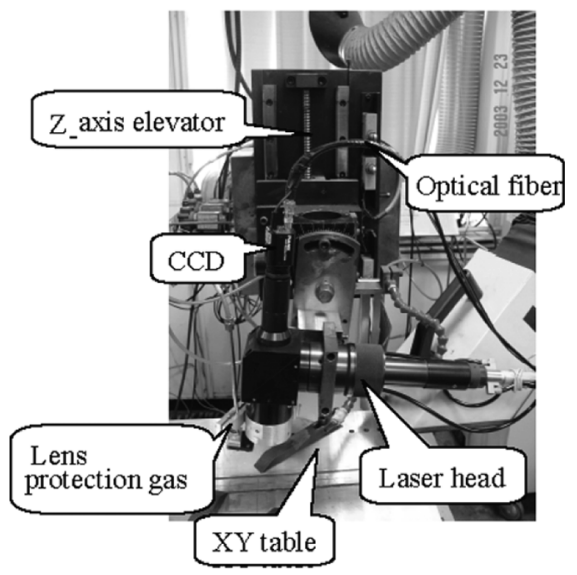

(b)

Fig. 2. (a) A 2.5-kW Nd-YAG laser (Rofin Sinar Inc.); (b) A laser head mounted on the XYZ table.

process. Due to the difference in wavelength, we can draw the following conclusion. For metals and carbides, the absorptance decreases with increasing wavelength. On the contrary, for oxides and polymers, the powder absorptance increases with increasing wavelength. In addition, when the laser starts heating the powder, the absorptance rises quickly. But for a higher power density, the melting of particles occurs obviously. Then it is followed by a drastic reduction in porosity, so the absorptance is decreased [13].

As shown in Fig. 2(a), a 2.5-kW Rofin Sinar Nd-YAG laser is used in our RT process. As shown in Fig. 2(b), a laser head is mounted on the XYZ table. The laser light can be transferred to the laser head through the optical fiber. The shielding gas (nitrogen) can protect the molten metal from oxygenation and keep the lens clean. A powder feeder with a lateral nozzle can convey metallic powder to the molten pool.

\section{B. Metallic Powder Feeder System}

The powder feeder is a self-contained unit designed to deliver powder at a precise rate to the powder nozzle. As shown in Fig. 3, the powder feeder is comprised of a hopper assembly, control cabinet, a control panel, a carrier gas filter assembly, an air vibrator, and a powder port shaft. The metallic powder feeding system utilizes gravity, fluidization, pressure differential, vibrational energy, and an inert carrier gas to deliver powder at a consistent and controlled feed rate.

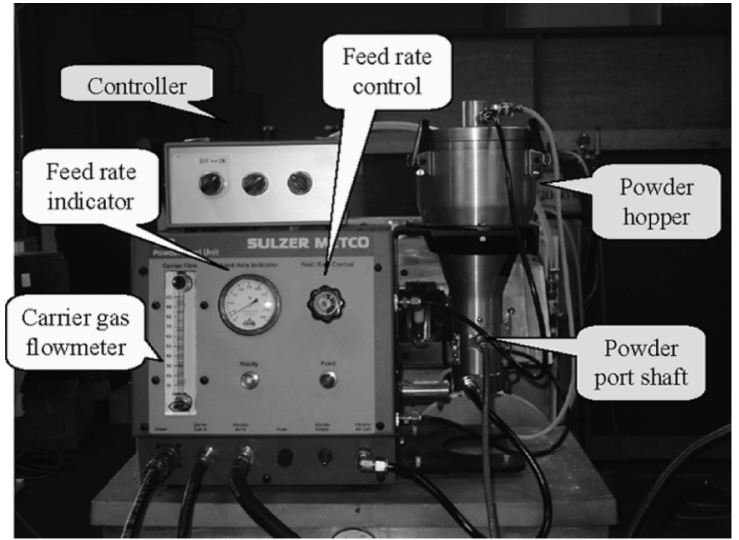

Fig. 3. Actual metallic powder feeder system.

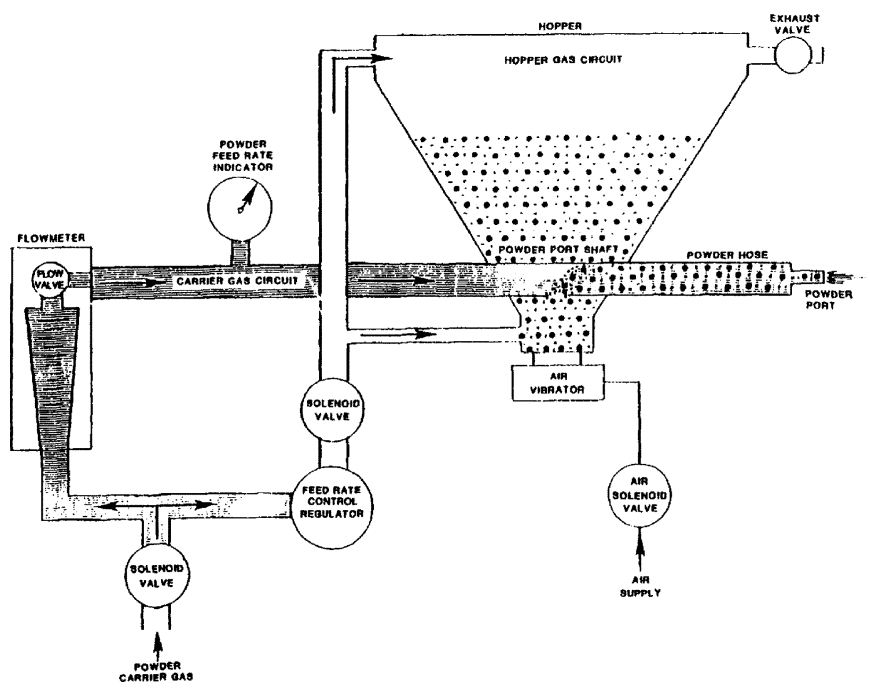

Fig. 4. Operation diagram of the powder feeder.

As shown in Fig. 4, the rate at which fluidized powder is injected into the powder port shaft varies directly with the pressure of the hopper gas. Therefore, the pressure regulator, which initially reduces the hopper gas pressure, is also used to control the powder feed rate. It is called the feed rate control regulator. The powder carrier gas flows through the powder port shaft where it entrains the powder being injected by the hopper gas, and carries it forward through the powder feed hose. The hopper gas pressure controls the rate at which powder is injected into the powder port shaft; the higher the hopper pressure setting, the higher the feed rate.

The performance of the powder feeder is evaluated by several experiments. According to the experimental results, the carrier gas flow rate is set to $3.8 \mathrm{l} / \mathrm{min}$. Once set, the flow of carrier gas will not change unless incoming gas pressure changes. The results of total powder weight with different feed rates are shown in Fig. 5. The feed rates are represented by the hopper pressure. In Fig. 5, the feed rate of 25 (psi) is too low to entrain the powder from the hopper. As a result, the total powder weight is zero. The experiments are repeated three times and recorded. The repeatability and the linearly of the powder feeder are good because the total weight of the powder increases linearly over time. 


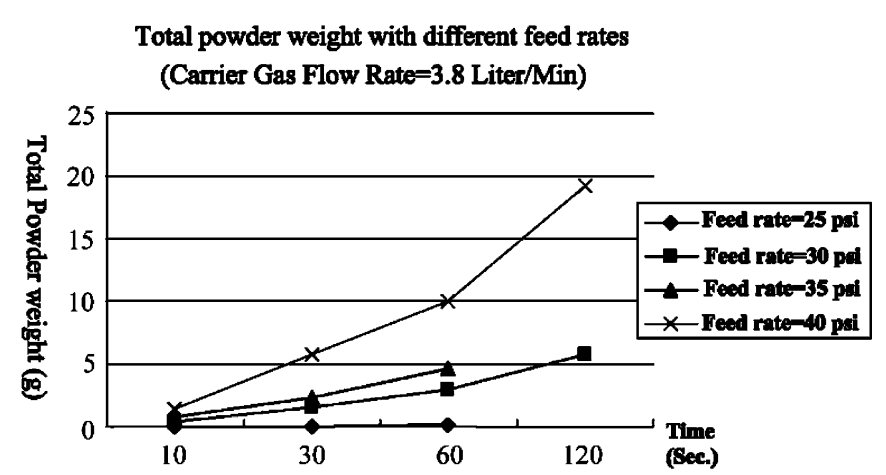

Fig. 5. Total powder weight with different powder feed rates.

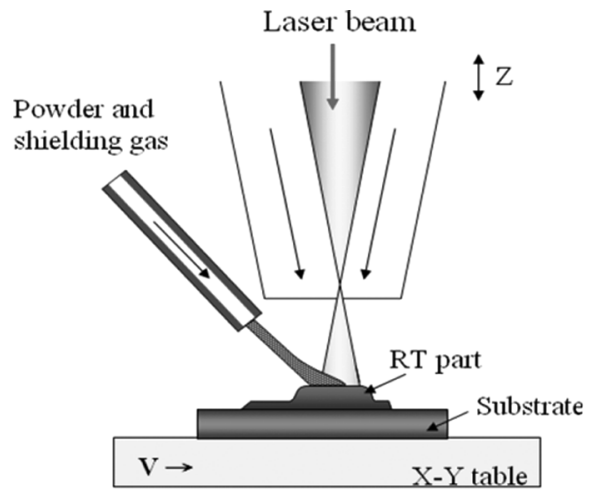

(a)

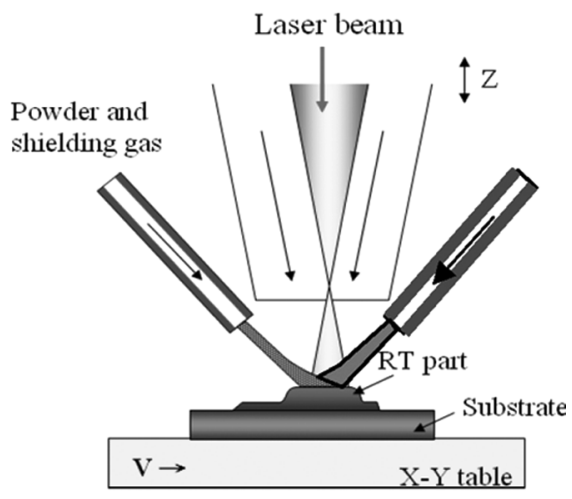

(b)

Fig. 6. (a) Single lateral powder nozzle. (b) Dual lateral powder nozzles.

\section{Powder Nozzle System}

The lateral powder nozzle is adopted for the proposed system. The angle between powder flow and horizontal plane was fixed on $60^{\circ}$. In order to guarantee the cladding conditions of each layer are the same, the focusing laser beam and the powder nozzle are aligned. They are raised a distance equal in height of a single layer after each layer is completed. For a lateral supply powder nozzle, the formation of the cladding is dependent on the direction of the powder delivery and the workpiece movement. As shown in Fig. 6, the single and dual lateral powder nozzles are used in this research to compare the quality of RT parts.

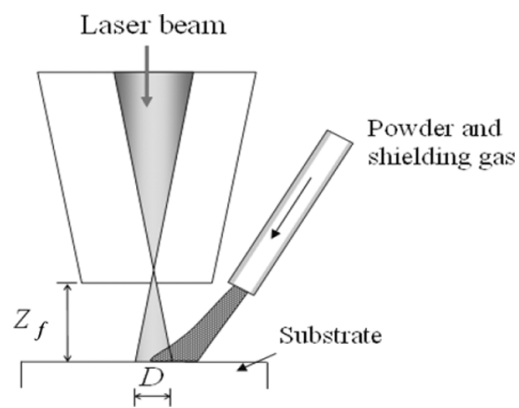

Fig. 7. Relation of the spot size $(D)$ and the distance from the focal point of the lens to the substrate $\left(Z_{f}\right)$.

\section{Shielding Gas-Supply System}

The shielding gas can affect the formation of plasma which may block the laser beam and affect its absorption into the workpiece. The formation of plasma is thought to occur through the reaction of the hot metal vapors from the melting pool with the shielding gas. The plasma formed above the melting pool with the shielding gas will be absorbed to an extent determined by the temperature and the ionization potential of the gases involved. The ionization potential of the shielding gases is often encountered in laser processing. The plasma blocking effect will be less for those gases having a high ionization potential. Despite the cost, helium is the best shielding gas in laser cladding. The nitrogen gas we use has a higher ionization potential to prevent the formation of plasma and the oxygenation of the metallic part for the proposed system [14]. There is another important consideration for laser cladding. When the flow of shielding gas is small, the powder stream is scattered and, thus, the amount of powder injected into the molten pool will be small. With an increase of the shielding gas, the powder stream is successfully restrained and narrowed, and the amount of the powder fed into the molten pool is increased.

\section{CRitical Parameters Influencing THE QUALITY OF THE RT PARTS}

\section{A. Important Parameters of the Direct Metallic RT System}

The process parameters for the direct metallic RT system include the melting time, traverse speed, laser spot size, etc. The relations between these parameters are as follows [15]:

$$
D=\frac{D_{b} Z_{f}}{f}
$$

where $D$ is the laser spot size (in millimeters), $D_{b}$ is the laser beam diameter (in millimeters), $Z_{f}$ is the distance from the focal point of lens to the substrate (in millimeters), and $f$ is the focal length of the focusing lens (in millimeters) (see Fig. 7). The laser spot size $(D)$ increases quickly when increasing the distance of $Z_{f}$

$$
E=\frac{P}{D v}
$$

where $E$ is the specific energy $\left(\mathrm{J} / \mathrm{mm}^{2}\right), v$ is the traverse speed $(\mathrm{mm} / \mathrm{s})$, and $P$ is the laser power (in watts). 


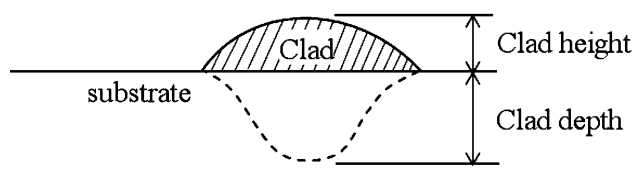

Fig. 8. Schematic diagram of clad height and depth.

The relationship between the laser spot size and the distance stray from the focus is defined as

$$
Z_{f}=\frac{0.32 \pi}{\lambda} D^{2}
$$

where $\lambda$ is the wavelength of the laser.

We also find the relation between melting time and mass by the use of the following equation to acquire the reference data for practical experiment:

$$
E=C \alpha m \Delta T
$$

where $C$ is the specific heat $\left(\mathrm{kcal} / \mathrm{kg}^{\circ} \mathrm{C}\right), \alpha$ is a transfer coefficient ( $1 \mathrm{kcal} / 4186 \mathrm{~J}$ ), $m$ is the mass (in kilograms), and $\Delta T$ is the temperature difference $\left({ }^{\circ} \mathrm{C}\right)$

$$
T_{m}=\frac{E}{P}
$$

where $T_{m}$ is the melting time (in seconds).

There are two main problems in laser cladding: the penetration of the substrate and the inability to melt the metallic powder entirely. An uneven distribution of the temperature is the cause of unmelted powder particles in the molten pool due to lower laser power. The phenomenon causes porosity and poor mechanical properties [16]. Specific energy determines the maximum layer thickness during the laser cladding. If the specific energy is too low, the bond between the substrate and the clad layer cannot be easily formed. On the other hand, if the specific energy is too powerful, the dilution will become too large and cause the previous cladding layer to melt down and residual stresses may be induced upon solidification. The dilution mainly depends on specific energy. A larger laser beam spot size will decrease the height of the cladding layer and increase the width if it has enough power density to melt metallic powder.

Dilution can be defined mathematically as the ratio of the clad depth in the substrate to the sum of clad height and clad depth (Fig. 8). It determines the amount of the liquid layer that needs to be formed on the substrate to ensure proper layer bonding. There are several parameters to determine the amount of dilution, such as powder-flow rate, laser power delivered to the substrate, and $\mathrm{X}-\mathrm{Y}$ table traverse speed, etc.

\section{B. Design of RT Experiments}

1) Taguchi's Method: Taguchi's method [17] has been used widely to optimize both process design and product design. For a robust design, Taguchi's method must reduce product variation by reducing the sensitivity of the product to the sources of variation rather than by controlling these sources. The primary advantage of Taguchi's method is that the design of parameters can be scientifically analyzed.
TABLE I

List of PROCESS PARAMETERS AND THEIR LEVELS

\begin{tabular}{|l|c|c|c|}
\hline Process parameters & Level 1 & Level 2 & Level 3 \\
\hline A: Laser Power (W) & 600 & 900 & 1200 \\
\hline B: Scanning speed (mm/min) & 300 & 600 & 900 \\
\hline C: Nozzle offset $\Delta X(\mathrm{~mm})$ & -1 & 0 & 1 \\
\hline D: Powder feed rate (g/min) & 8 & 10 & 12 \\
\hline E: Laser spot size (mm) & 2 & 3 & 4 \\
\hline F: Tool path offset (mm) & 0.25 & 0.5 & 0.75 \\
\hline
\end{tabular}

The signal-to-noise ratio (SNR) measures the sensitivity of the quality characteristic being investigated to those uncontrollable external factors. That is, the SNR is an objective index to measure the stability of quality. If the SNR is larger, the quality of the experiment becomes better with this combination of factors and levels. There are three types of SNRs:

1) lower is better (L.B.);

2) nominal is best (N.B.);

3) higher is better (H.B.).

Generally, the inaccuracy of RP/RT parts seldom happens in the $\mathrm{X}$ and $\mathrm{Y}$ directions. The $\mathrm{Z}$-axis dimension of the part may be the major source of dimensional inaccuracy because of improper process parameters. The N.B. analysis model is used in this study. The target dimension of the layer thickness is $1 \mathrm{~mm}$ in the Z-axis. The equations of the N.B. are shown as follows:

$$
\begin{aligned}
\frac{S}{N_{N B}} & =10 \log \left[\frac{S_{m}-V_{e}}{n V_{e}}\right] \\
\text { Sensitivity(S) } & =10 \log \left[\frac{S_{m}-V_{e}}{n}\right]
\end{aligned}
$$

where

$$
\begin{gathered}
S_{m}=\text { Sum of square due to the mean }=\frac{T^{2}}{n} \\
V_{e}=\text { Variance due to error }=\frac{\sum y_{i}^{2}-S_{m}}{n-1}, \\
\quad y_{i} \text { is the } i \text { th export (output) value } \\
n \text { is the number of the data } \\
T=\text { Sum of all the data }=\sum_{i=1}^{n} y_{i} .
\end{gathered}
$$

2) Selection of Process Parameters: The objective of the study is to analyze the effect of process parameters on the layer thickness of the RT part produced by the proposed RT process. The process parameters for the proposed RT system include: laser power, scanning speed, nozzle offset $\Delta X$, powder feed rate, laser spot size, and tool path offset. The process parameters and the levels chosen for the Taguchi's experiments are listed in Table I. The relation to the process parameters for the proposed system is shown in Fig. 9. 


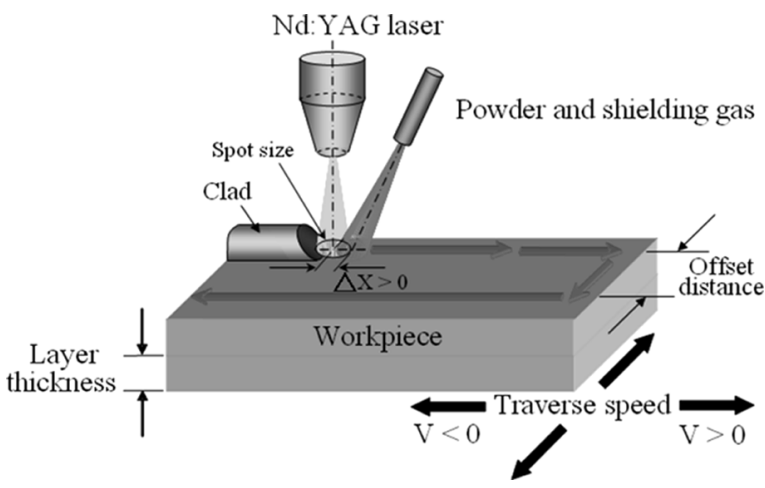

Fig. 9. Relation to the process parameters for the proposed system.

TABLE II

RESPONSE TABLE AND LAYER THICKNESS

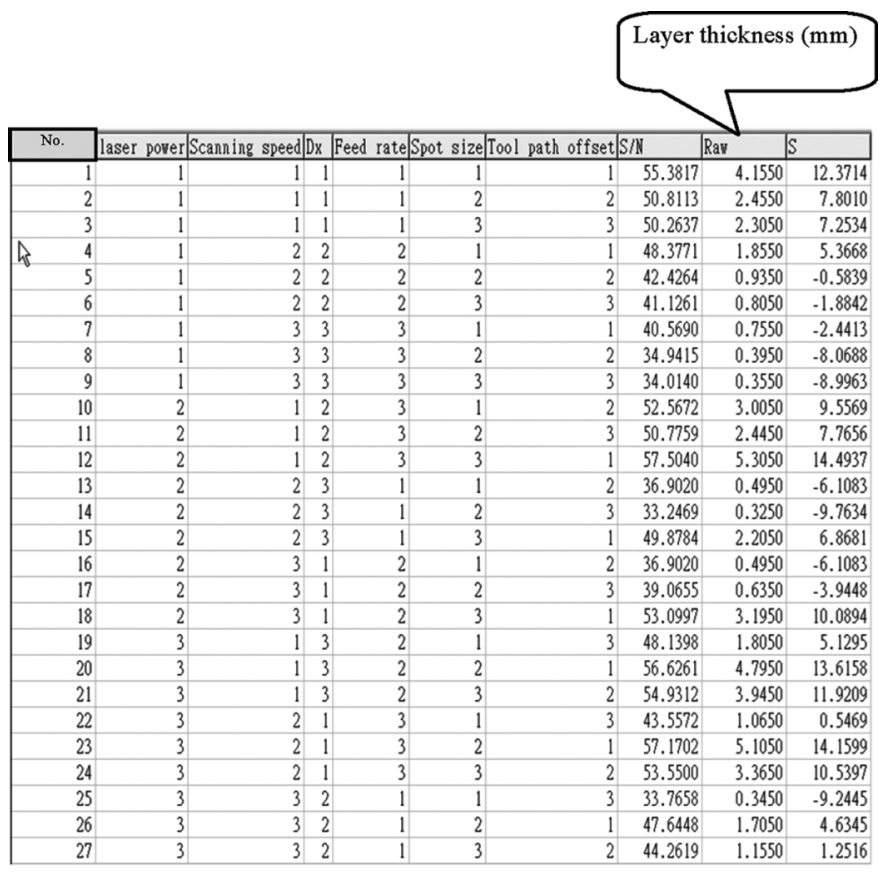

The nozzle offset $(\Delta X)$ is defined as the distance between the center of the spot size and the intersection point of the axis of the powder stream and substrate surface. $\Delta X$ is defined as positive when the intersection point of the axis of the powder stream and laser beam is beneath the center in the spot size; otherwise, it is negative. An orthogonal array can be constructed from the design parameters, their levels, and the specific interactions among the design parameters. In this study, a L27 orthogonal array (Table II) with 6 columns and 27 rows is chosen. Each design parameter has three levels assigned to each column of the array. The 27 rows represent the 27 experiments to be conducted.

\section{Experimental Results and Analysis}

Based on the N.B. model of the Taguchi's method, the SNR [see (6)-(10)] is a summary statistic representing the ratio of mean $(\mu)$ and variance $(\sigma)$. The sensitivity $(S)$ adjusts the mean of the output quality values to the target value. These two values

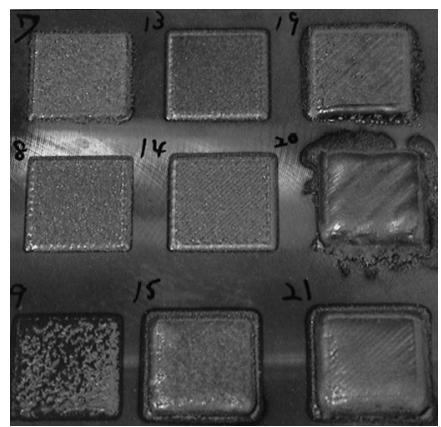

Fig. 10. Experimental results for determining the layer thickness with different process parameters.

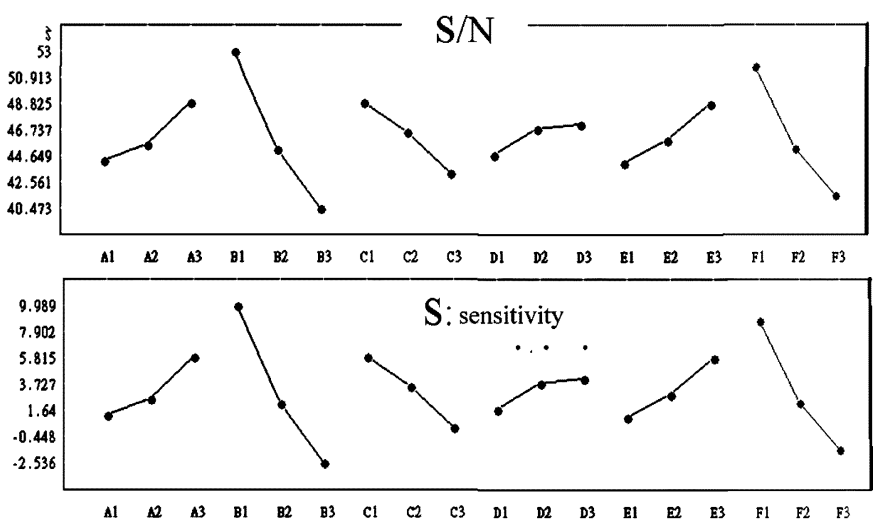

Fig. 11. Response graph of the experimental results.

are the way to determine the significant factors in the optimization process [18].

Fig. 10 is the experimental result for determining the layer thickness with different process parameters. With different process conditions, the square-shaped test parts in Fig. 10 result in different layer thicknesses. The target dimension of the layer thickness is $1 \mathrm{~mm}$ in the Z-axis. The layer thickness results are listed in Table II. The calculated N.B. SNRs for each experiment are also listed in Table II. Based on the experimental results, a response table (Table II) and response graph (Fig. 11) are derived for the control factors of each experiment. From Table II or Fig. 11, we choose the factors with the greatest SNR as the most optimal process parameters combination, which are $\mathrm{A}_{3} \mathrm{~B}_{1} \mathrm{C}_{1} \mathrm{D}_{3} \mathrm{E}_{3} \mathrm{~F}_{1}$. The recommended value of the process parameters is shown as follows:

- laser power $=1200 \mathrm{~W}$;

- scanning speed $=300 \mathrm{~mm} / \mathrm{min}$;

- nozzle offset $=-1 \mathrm{~mm}$;

- powder feed rate $=12 \mathrm{~g} / \mathrm{min}$;

- laser spot size $=4 \mathrm{~mm}$;

- tool path offset $=0.25 \mathrm{~mm}$.

ANOVA analysis [19] provides a significance rating of the various factors in this study. ANOVA is a useful technique for estimating error variance and for determining the relative importance of various design parameters. The results of the ANOVA computations are listed in Table III. From Table III, the contribution (P\%) of factors B (scanning speed) and F (tool path offset) are very large. The factors B (scanning speed) and F (tool path 
TABLE III

RESULTS OF ANOVA ANALYSIS

\begin{tabular}{|l|c|c|c|c|c|c|}
\hline \multicolumn{1}{|c|}{ S.O.V } & S.S. & D.F. & V & F & S' & P\% \\
\hline A: Laser Power & 4.8084 & 2 & 2.4042 & 10.88929 & 4.366829 & 6.752093 \\
\hline B: Scanning speed & 25.7876 & 2 & 12.8938 & 58.39961 & 25.34603 & $\mathbf{3 9 . 1 9 0 6 3}$ \\
\hline C: Nozzle offset & 3.4583 & 2 & 1.72915 & 7.831802 & 3.016729 & 4.664537 \\
\hline D: Powder feed rate & 2.4866 & 2 & 1.2433 & 5.631252 & 2.045029 & 3.162071 \\
\hline E: Spot size & 4.1921 & 2 & 2.09605 & 9.493594 & 3.750529 & 5.799156 \\
\hline F: Tool path offset & 20.8496 & 2 & 10.4248 & 47.21682 & 20.40803 & $\mathbf{3 1 . 5 5 5 3 8}$ \\
\hline e (error) & 3.091 & 14 & 0.220786 & & 5.740529 & 8.876141 \\
\hline T (Sum) & 64.6737 & 26 & & & 64.6737 & 100 \\
\hline where
\end{tabular}

SOV: Source of Variation

S.S.: Sum of Squares

D.F.: Degrees of Freedom

V: Variance

$\mathrm{F}: \mathrm{F}=\mathrm{Vi} / \mathrm{Ve}$

$\mathrm{S}^{\prime}=$ S.S.-( D.F.-1)*Ve

$\mathrm{P} \%=$ The percent of the contribution

offset) are the most significant factors affecting the layer thickness of RT parts.

\section{COMParison OF Single AND Dual LATERAL POWDER NOZZLE}

The purpose of the lateral powder nozzle is to deliver the metallic powder to the molten pool on the formation of the cladding. The single lateral powder nozzle is shown in Fig. 12(a). With the single lateral powder nozzle, the formation of the cladding is strongly dependent on the direction of the powder delivery and the RT part movement. In order to solve this problem, the dual lateral powder nozzle [Fig. 12(b)] was designed to feed the metallic powder from both sides of the laser beam. With the dual nozzle system, the metallic powder could be distributed more uniformly than the single nozzle system. As shown in Fig. 13(a), the metallic cube is produced by the proposed RT system with a single lateral nozzle. From Fig. 13(a), the height of the metallic cube is not uniform. However, the height of the RT parts manufactured by the dual lateral nozzle system [Fig. 13(b)] is more uniform. From Fig. 13(b), we can see the dimensional accuracy of the RT parts is better than the metallic cube in Fig. 13(a).

\section{Build Injection Mold With the Direct METALLIC RT SYSTEM}

\section{A. Fabrication of the Injection Mold}

RT is capable of generating core and cavity inserts as a product of an RP technology. RT is the result of an additive process driven by 3-D CAD-a process that requires little or no machining or electric discharge machining (EDM). The advantages of the direct metallic RT system include: shortening of the tooling lead time; reduced cost; functional test of parts

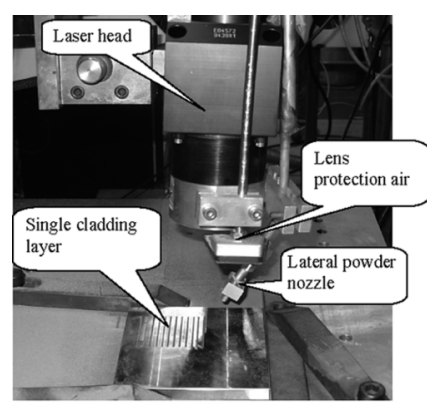

(a)

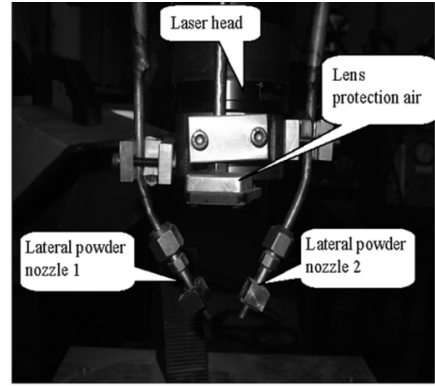

(b)
Fig. 12. (a) Single lateral powder nozzle. (b) Dual lateral powder nozzle.

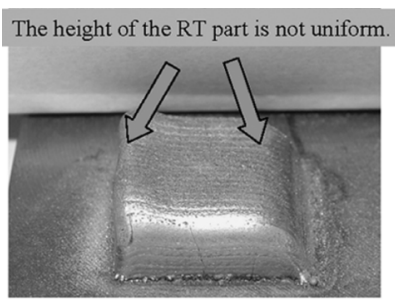

(a)

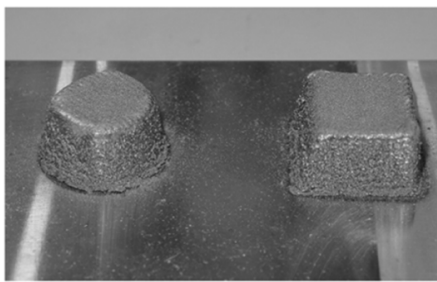

(b)
Fig. 13. (a) Metallic cube produced by the RT system with a single lateral powder nozzle. (b) The RT parts produced by the RT system with a dual lateral powder nozzle.

in early design becomes possible; and an ability to directly transfer CAD data.

Fig. 14 shows a flowchart of the development of the injection mold with the direct metallic RT system. Generating a CAD model [Fig. 15(a)] of mold inserts (core and cavity) for a computer mouse part is the first step in this process. As shown in Fig. 15(b), the CAD model can be transferred to the tessellated model (stereolithography (STL) file). The tessellated model is produced with a height of $24 \mathrm{~mm}, 4210$ facets. The STL file is loaded and sliced by using the preprocess RT software [20] developed by our lab at the Intelligent Automation Laboratory at National Chung Cheng University. The sliced result (layer thickness $=1 \mathrm{~mm}$, total layer number $=24$ ) is shown in Fig. 16. The RT software continues to generate the tool paths (Fig. 17) after the slicing process. The tool paths are transferred to the machine control code. This machine control code is then transferred to the PC-based RT machine controller which can control the $\mathrm{X}-\mathrm{Y}$ working table to move with the tool path.

As shown in Fig. 18, the mold insert is manufactured by the proposed direct RT system with a dual powder nozzle. Layer by layer, the mold insert (core) produced by the proposed RT system is shown in Fig. 19. In order to save manufacturing time, the layer thickness is set as $1 \mathrm{~mm}$. The staircase effect is clear on the surface of the mold insert. If we use the thinner layer thickness to produce the mold insert, the staircase effect is greatly improved. However, the fabrication time is increased. As shown in Fig. 20, the mold insert is finished with a CNC milling machine to diminish the staircase effect. It takes $6 \mathrm{~h}$ to produce the mold core (Fig. 19) by the proposed RT system. The CNC milling machine needs $1 \mathrm{~h}$ to finish the mold core (Fig. 20).

At the beginning of RT part fabrication, the substrate should be preheated to minimize the residual thermal stress. For the 


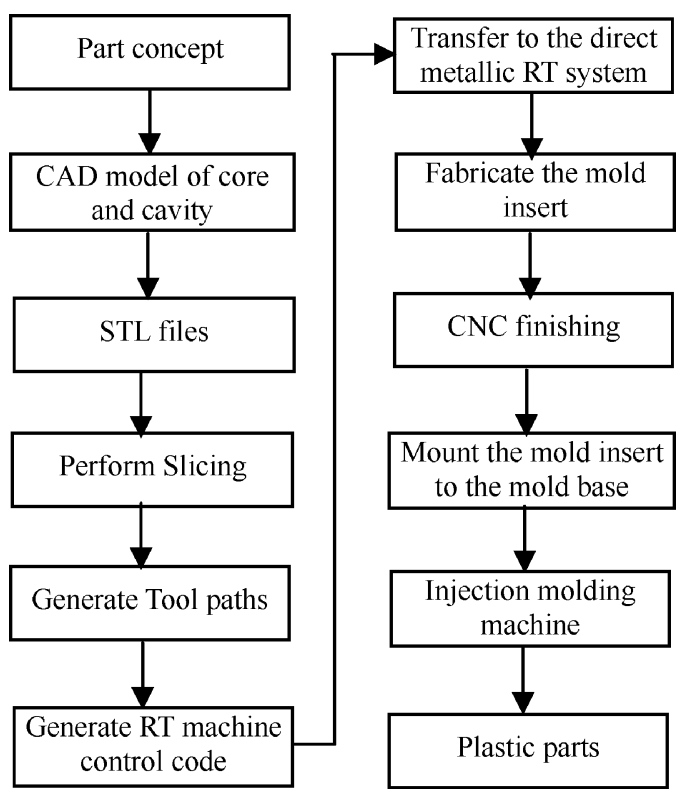

Fig. 14. Flowchart of the development of the injection mold with the direct metallic RT system.

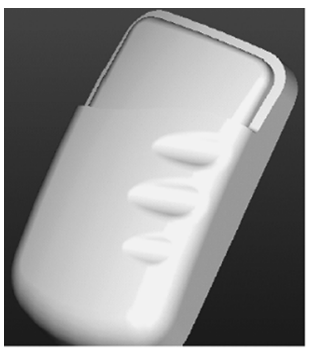

(a)

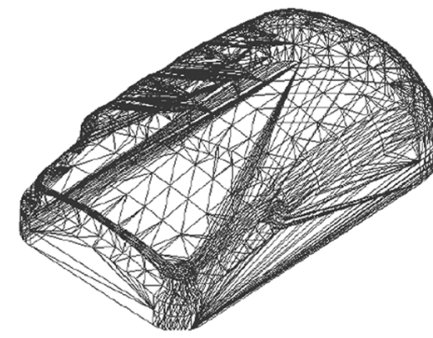

(b)
Fig. 15. (a) CAD model of the mold insert. (b) The tessellated model (STL file) of the mold insert.

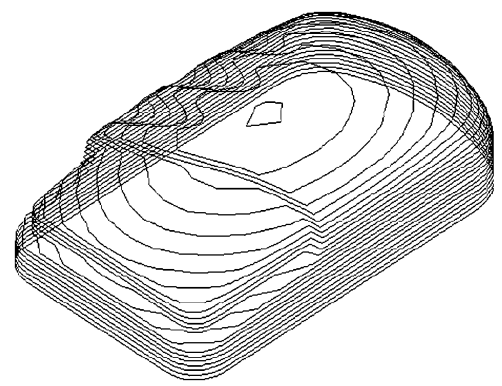

Fig. 16. Sliced result (layer thickness $=1 \mathrm{~mm}$, total layer number $=24$ ) of the mold insert.

first or second layers, we should increase the laser power and decrease the feed rate of the metallic powder. With more laser power and less metallic powder, the first or second layers could be firmly bonded to the substrate. Otherwise, a protrusion phenomenon (Fig. 21) could be found on the boundary of the RT part and the substrate.

\section{B. Material Testing of the Injection Mold}

1) Hardness Testing: The material testing is very important for the RT parts. Among these material properties, hardness is an index to measure the life of the mold inserts. In this research, the Rockwell hardness tester is used to measure the hardness

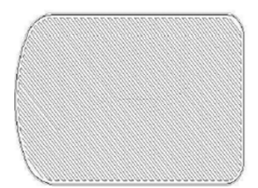

Layer 1

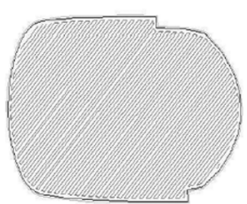

Layer 16

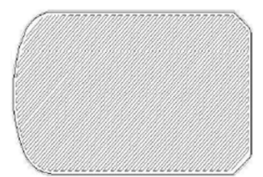

Layer 8

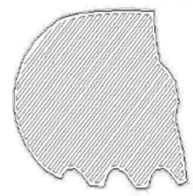

Layer 20

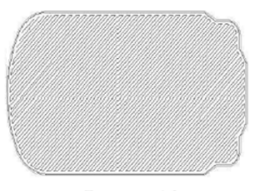

Layer 10
Fig. 17. Tool paths of different layers.

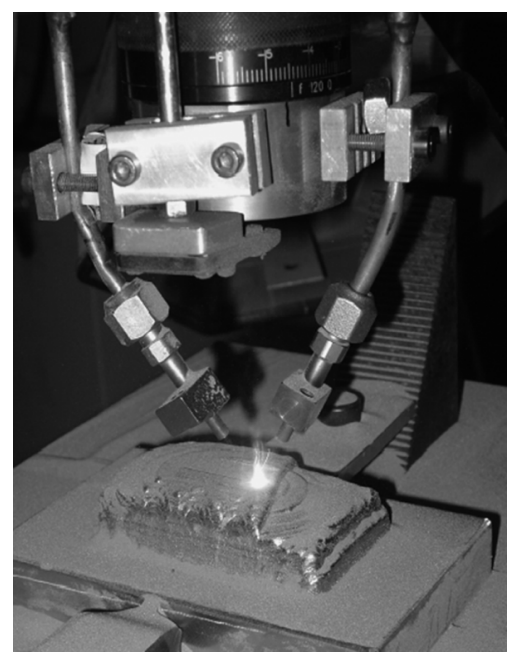

Fig. 18. Mold insert is manufactured by the direct RT system with a dual powder nozzle.

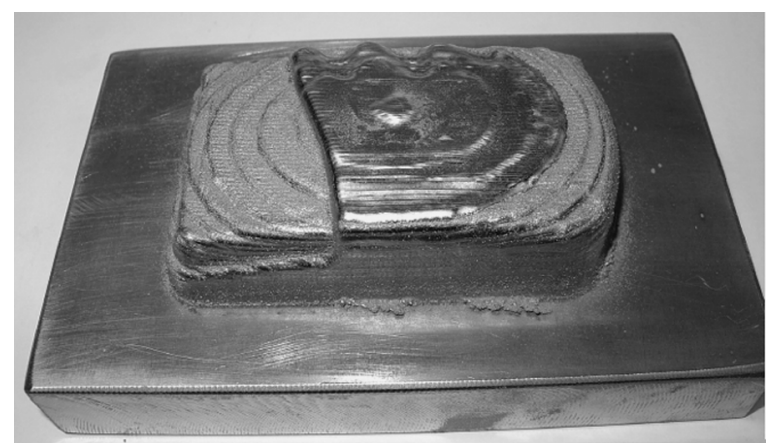

Fig. 19. Mold insert (core) produced by the proposed RT system.

of the mold inserts. As shown in Table IV, the tested value of hardness (HRC) is 25-28 for the mold insert. The HRC for the general mold steel (Tempering steel, 2311 ISO-BM) is 29-34. From these testing results, the hardness of the mold insert is close to the general mold steel.

2) Microstructure of RT Parts: Fully dense material is needed for RT part fabrication to achieve optimum mechanical strength. Full density is achieved by optimization of both process parameters and motion path to maintain a continuous molten pool that sweeps the entire volume of the RT part. Porous microstructures result primarily from gas evolution 


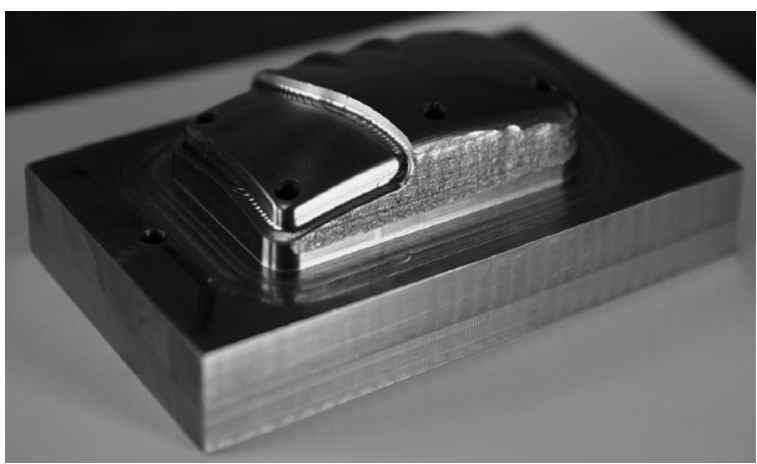

Fig. 20. Finished mold insert after CNC surface finishing.

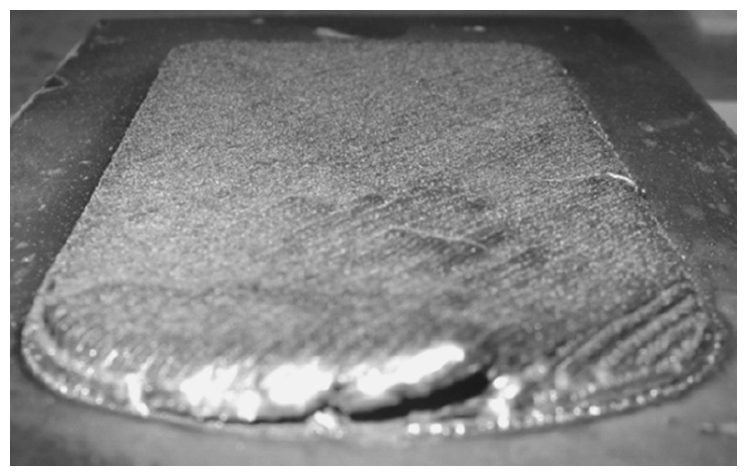

Fig. 21. Protrusion phenomenon of the clad layer.

TABLE IV

ROCKWELl HARDNESS TESTING OF THE MOLD INSERT

\begin{tabular}{|c|c|c|}
\hline & Substrate & RT mold \\
\hline Material & $\begin{array}{c}\text { Tempering steel } \\
(2311 \text { ISO-BM) }\end{array}$ & $\begin{array}{c}\text { Nickel based alloy powder } \\
\text { (Colmonoy 43) }\end{array}$ \\
\hline $\begin{array}{c}\text { Rockwell Hardness } \\
\text { (HRC) }\end{array}$ & $29-34$ & $25-28$ \\
\hline
\end{tabular}

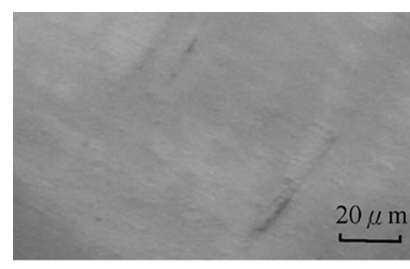

(a)

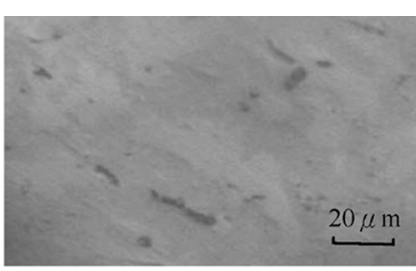

(b)
Fig. 22. (a) Fully dense microstructure of an RT part. (b) The porous microstructure results primarily from gas evolution during solidification.

during solidification and lack of fusion between layers or adjacent passes of the molten pool [16].

The process settings that yielded the results reported in Figs. 22 and 23 are listed in Table V, where the following process parameters are kept constant:

1) scanning speed $=300 \mathrm{~mm} / \mathrm{min}$;

2) nozzle offset $\Delta X=-1 \mathrm{~mm}$.

The microstructure study was performed on the cross sections of the RT parts. After metallographically preparing the cross sections to mirror finish, the specimens were etched with an acid

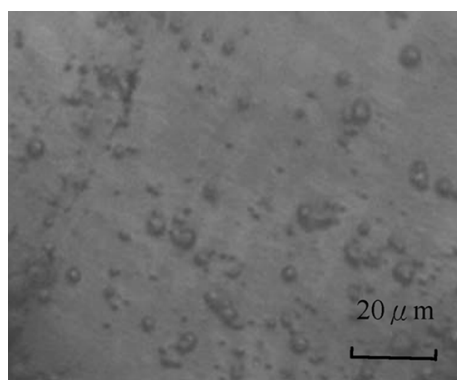

Fig. 23. Microstructure of nonmelted powder particles is formed at low-power density.

TABLE V

PROcess SetTings That YieLded the Results RePORTED IN Figs. 22 AND 23

\begin{tabular}{|c|c|c|c|c|}
\hline & $\begin{array}{c}\text { Laser power } \\
(\mathrm{W})\end{array}$ & $\begin{array}{c}\text { Powder feed } \\
\text { rate }(\mathrm{g} / \mathrm{min})\end{array}$ & $\begin{array}{c}\text { Laser spot } \\
\text { size }(\mathrm{mm})\end{array}$ & $\begin{array}{c}\text { Tool path } \\
\text { offset }(\mathrm{mm})\end{array}$ \\
\hline Fig. 22(a) & 1200 & 10 & 4 & 0.25 \\
\hline Fig. 22(b) & 1200 & 12 & 4 & 0.5 \\
\hline Fig. 23 & 600 & 12 & 3 & 0.25 \\
\hline
\end{tabular}

where the following process parameters are kept constant:

(1) Scanning speed $=300 \mathrm{~mm} / \mathrm{min}$

(2) Nozzle offset $\Delta X=-1 \mathrm{~mm}$

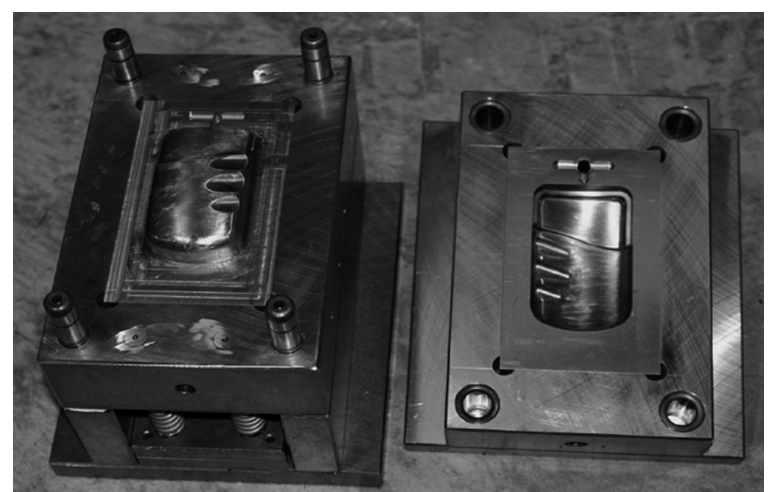

Fig. 24. Core and cavity of the direct metallic RT mold.

solution and examined under an optical microscope. Fig. 22(a) shows the fully dense microstructure of an RT part. As shown in Fig. 22(b), the porous microstructure results primarily from gas evolution during solidification. The porous microstructure is formed during melting and remains in the solidified microstructure. In Fig. 23, the microstructure of nonmelted powder particles is formed at low-power density.

\section{Injection Molding Process of the Direct Metallic RT Mold}

One of the most common methods for shaping plastic material is a process called injection molding. After finishing the mold inserts (core and cavity), the designed gate, sprue, and runner are manufactured on the mold insert. The completed mold inserts are mounted to the standard mold base for assembling the whole injection mold (Fig. 24). The injection mold is mounted to the injection molding machine. After tuning the process parameters of the injection molding process, the resulting acrylonitrile butadiene styrene (ABS) plastic part is generated as shown in Fig. 25. 

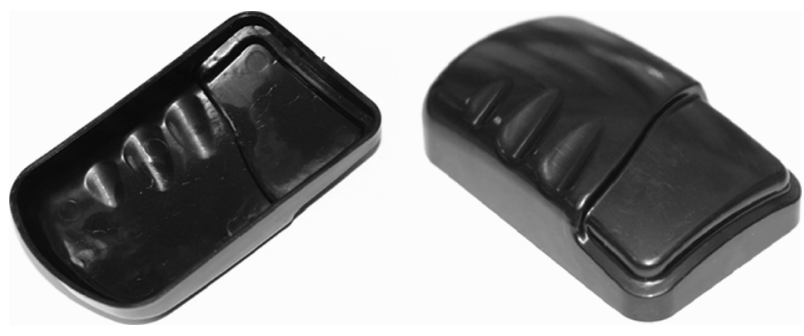

Fig. 25. ABS plastic part produced by the injection molding machine.

\section{CONCLUSION}

In this paper, the direct metallic RT system has been demonstrated. The hardware architecture (a 2.5-kW Nd-YAG laser system, a metallic powder feeder system, a powder nozzle system, and a shielding gas-supply system) has been introduced, respectively. By using Taguchi's method, the critical process parameters influencing the dimensional accuracy are analyzed. From the ANOVA computational results, the factors "scanning speed" and "tool path offset" are the most influential factors affecting the layer thickness of RT parts.

From the experimental results, the dimensional accuracy of the dual nozzles RT system is better than the single-nozzle RT system. Finally, the proposed RT system can be extended to fabricate the mold inserts. After finishing the mold inserts and assembling the whole injection mold, the injection molding machine can generate the ABS plastic parts with excellent quality.

The following recommendations for future applications of the proposed RT system are listed.

1) For functional consideration, the RT system can produce a functional grading RT part with multiple metallic powders.

2) The temperature or pressure sensors can be embedded into the mold insert with RT technology, so that it can measure the actual temperature and pressure inside the core and cavity during the molding process

\section{REFERENCES}

[1] E. Sachs, M. Cima, P. Williams, D. Brancazio, and J. Cornie, "Threedimensional printing: Rapid tooling and prototypes directly from a CAD model," Ann. CIRP, vol. 39, no. 1, pp. 201-204, 1990.

[2] V. M. Weerasinghe and W. M. Steen, "Laser cladding by powder injection," in Proc. 1st Int. Conf. Lasers in Manufacturing, 1983, pp. 125-132.

[3] E. W. Kreutz, G. Backes, A. Gasser, and K. Wissenbach, "Rapid prototyping with $\mathrm{CO}_{2}$ laser radiation," Appl. Surf. Sci., vol. 86, pp. 310-316, 1995.

[4] D. M. Keicher, W. D. Miller, J. E. Smugeresky, and J. A. Romero, "Laser engineered net shaping (LENS): Beyond rapid prototyping to direct fabrication," in Proc. TMS Annu. Meeting, San Antonio, TX, 1998, pp. 369-377.

[5] R. Merz, F. B. Prinz, K. Ramaswami, M. Terk, and L. E. Weiss, "Shape deposition manufacturing," in SFF Symp., Austin, TX, 1994.

[6] J. O. Milewski, D. J. Thoma, J. C. Fonseca, and G. K. Lewis, "Development of a near net shape processing method for rhenium using light fabrication," J. Mater. Manuf. Process., vol. 13, no. 5, pp. 719-730, 1998.

[7] J. Y. Jeng, S. C. Peng, and C. J. Chou, "Metal rapid prototype fabrication using selective laser cladding technology," Int. J. Adv. Manuf. Technol., vol. 16, pp. 681-687, 2000.

[8] J. Mazumder, A. Schifferer, and J. Choi, "Direct materials deposition: Designed macro and microstructure," Mat. Res. Innovations, vol. 3, pp. 118-131, 1999.
[9] M. R. Boddu, R. G. Landers, and F. W. Liou, "Control of laser cladding for rapid prototyping-A review," in Proc. Solid Freeform Fabrication, 2001, pp. 460-467.

[10] P. A. Vetter, T. Engel, and J. Fontaine, "Laser cladding: The relevant parameters for process control," in Proc. Int. Soc. Opt. Eng., 1994, vol. 2207, pp. 452-462.

[11] R. C. Luo, C. L. Chang, J. H. Tzou, and Z. H. Huang, "Automated desktop manufacturing: Direct metallic rapid tooling system," in Proc. IEEE Int. Conf. Robotics and Automation, Barcelona, Spain, Apr. 18-22, 2005.

[12] R. C. Luo, C. C. Chen, and J. H. Tzou, "The development of a direct metallic rapid prototyping system," in Proc. IEEE Int. Conf. Robotics and Automation, New Orleans, LA, Apr. 26-May 1, 2004.

[13] K. Tolochko, T. Laoui, V. Khlopkov, and E. Mozzharov, "Absorptance of powder materials suitable for laser sintering," Rapid Prototyping J., vol. 6, no. 3, pp. 155-160, 2000.

[14] W. M. Steen, Laser Material Processing. New York: SpringerVerlag, 1991, ch. 4, pp. 121-122.

[15] R. Boddu, S. Musti, G. Landers, S. Agarwal, and W. Liou, "Empirical modeling and vision based control for laser aided metal deposition process," in Proc. Solid Freeform Fabrication, 2001, pp. 452-459.

[16] _ - "Empirical modeling and vision based control for laser aided metal deposition process," in Proc. Solid Freeform Fabrication, 2001, pp. 452-459.

[17] R. K. Roy, Design of Experiments Using the Taguchi Approach: 16 Steps to Product and Process Improvement. New York: Wiley, 2000.

[18] A. W. L. Yao and Y. C. Tseng, "A robust process optimization for a powder type rapid prototyper," Rapid Prototyping J., vol. 8, no. 3, pp. 180-189, 2002.

[19] H. R. Lindman, Analysis of Variance in Experimental Designs. New York: Springer-Verlag, 1992.

[20] R. C. Luo and J. H. Tzou, "The development of an intelligent webbased rapid prototyping manufacturing system," IEEE Trans. Autom. Sci. Eng., vol. 1, no. 1, pp. 4-13, Jul. 2004.

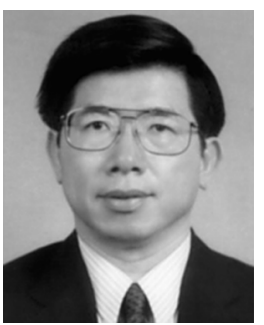

Ren C. Luo (F'92) received the Ph.D. degree in electrical engineering from the Technische Universitat Berlin, Berlin, Germany, in 1982.

Currently, he is a Professor in the Department of Electrical Engineering and the President of the National Chung Cheng University, Chia-Yi, Taiwan, R.O.C. His research interests include sensor-based intelligent robotic systems, multisensor fusion and integration, computer vision, rapid prototyping, and advanced manufacturing systems. He has authored many papers on these topics, which have been published in referred journals and conference proceedings. He is also the holder of several patents.

Dr. Luo was President of IEEE Industrial Electronics Society from 2000 to 2001 and President of the Chinese Institute of Automation Engineers. He is also the Technical Committee Chair on Multisensor Integration and Fusion of the IEEE Robotics and Automation Society. He is an Editor-in-Chief of IEEE/ASME TRANSACTIONS ON MECHATRONICS and Associate Editor of the IEEE TRANSACTIONS ON INDUSTRIAL ELECTRONICS. He received an ALCOA outstanding research award, IEEE Eugene Mittelman outstanding achievement award, and an outstanding research award from National Science Council in Taiwan. His professional society memberships include the IEEE Industrial Electronics Society and Robotics and Automation Society.

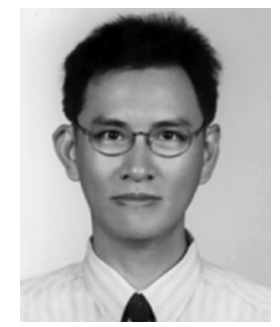

Jyh Hwa Tzou received the B.S. degree in mechanical engineering from National Cheng Kung University, Tainan City, Taiwan, R.O.C., in 1986, the M.S. degree in mechanical engineering from National Sun Yat-Sen University, Kaohsiung, in 1988, and the Ph.D. degree in electrical engineering from the National Chung Cheng University, Chia-Yi, in 2005.

Currently, he is an Associate Professor in the Department of Mechanical Engineering, Wu-Feng Institute of Technology, Chia-Yi. His research interests include rapid prototyping, rapid tooling, reverse engineering, and mechatronics systems. 